\title{
AUTHOR INDEX \\ VOLUME 15 (2001)
}

Ahn, S.J., Rauh, W. \& Kim, S.I., Circular coded target for automation of optical 3D-measurement and camera calibration

Arcelli, C. \& Serino, L., Regularization of graphlike sets in gray-tone digital images

Ayala, R., see Domínguez, E.

Bandyopadhyay, S., Maulik, U. \& Pakhira, M.K., Clustering using simulated annealing with probabilistic redistribution

Barber, K.S. \& Martin, C.E., Dynamic adaptive autonomy in multiagent systems: representation and justification

Basu, M. \& Su, M., Image smoothing with exponential functions

Bazzi, I., see Natarajan, P.

Ben-Arie, J. \& Wang, Z., Hierarchical shape description and similarity-invariant recognition using gradient propagation

Ben-Hamadou, A., see Chainbi, W.

Beveridge, J.R., see Draper, B.A.

Bhattacharya, P., see Huo, C.

Bloyet, D., see Elmoataz, A.

Bobick A.F., see Wilson, A.D.

Bowyer, K. \& Stark, L., Introduction: undergraduate education and computer vision

Brimkov, V.E., Optimally fast CRCW-PRAM testing 2D-arrays for existence of repetitive patterns

Briscoe, G., see Caelli, T.

Bui, H.H., Venkatesh, S. \& West, G., Tracking and surveillance in wide-area spatial environments using the abstract hidden Markov model

15 (2001) 905-919

15 (2001) 643-657

15 (2001) 1031-1052

15 (2001) 269-285

15 (2001) 405-433

15 (2001) $735-752$

15 (2001) 43-63

15 (2001) 1251-1261

15 (2001) 435-450

15 (2001) 823-831

15 (2001) 1299-1309

15 (2001) 1201-1212

15 (2001) 123-160

15 (2001) 753-755

15 (2001) 1167-1182

15 (2001) 197-221

Bui, T.D., see Feng, L.

Bui, T.D., Chen, G.Y. \& Feng, L., An orthonormal-shell-Fourier descriptor for rapid matching of patterns in image database

Bunke, H. \& Caelli, T., Editorial

Bunke, H., see Marti, U.-V.

Bunke, H., see $\mathrm{Yu}, \mathrm{K}$.

Caelli, T., see Bunke, H.

Caelli, T., McCabe, A. \& Briscoe, G., Shape tracking and production using hidden Markov models

Cattoni, R., see Charlton, P.

Cem Say, A. C., Understanding arithmetic problems in Turkish

Chainbi, W., Ben-Hamadou, A. \& Jmaiel M., A belief-goal-role theory for multiagent systems

Chang, M.-C., Fuh, C.-S. \& Chen, H.-Y., Fast search algorithms for industrial inspection

Charlton, P. \& Cattoni, R., Evaluating the deployment of FIPA standards when developing application services

15 (2001) 177-195

15 (2001) 329-345

15 (2001) 1213-1229

15 (2001) 1-3

15 (2001) 65-90

15 (2001) 161-176

15 (2001) 1-3

15 (2001) 197-221

15 (2001) 551-577

15 (2001) 359-374

15 (2001) 435-450

15 (2001) 675-690

15 (2001) 551-577

Chen, G.Y., see Bui, T.D.

15 (2001) 1213-1229

1331

International Journal of Pattern Recognition and Artificial Intelligence Vol. 15 No. 8 (2001) 1331-1335 (C) World Scientific Publishing Company 
Chen, H.-Y., see Chang, M.-C.

15 (2001) 675-690

Chen, L.-H., see Lee, K.-L.

Chen, L.-H., Deng, S.-H. \& Liao, H.-Y., MCE-based face recognition

Chen, Y. \& Okada, M., Structural analysis and semantic understanding for offline mathematical expressions

Chen, Y.Q., see He, H.

Choi, S.-M. \& Oh, I.-S., A segmentation-free recognition of handwritten touching numeral pairs using modular neural network

Daurat, A., Salient points of Q-convex sets

Deng, S.-H., see Chen, L.-H.

Ding, M., see Xiao, Y.

Doermann, D., see Guo, J.K.

Domínguez, E., Francés, A.R., Ayala, R. \& Quintero, A., A digital index theorem

Draper, B.A. \& Beveridge, J.R., Teaching image computation: from computer graphics to computer vision

Economou, G., see Makrogiannis, S.

Eickeler, S., see Müller, S.

Elmoataz, A., Schüpp, S. \& Bloyet, D., Fast and simple discrete approach for active contours for biomedical applications

Esteban, J.L., see Sanchez, A.

Fang, B., Wang, Y.Y., Leung, C.H., Tse, K.W., Tang, Y.Y., Kwok, P.C.K. \& Wong, Y.K., Offline signature verification by the analysis of cursive strokes

Feng, L., see Bui, T.D.

Feng, L., Bui, T.D. \& Tang, Y.Y., Classification of similar 2-D objects by wavelet-sparse-matrix (WSM) method

Feschet, F. \& Tougne, L., Generating isotropic discrete waves on cellular automata

Fink, E. \& Heath, M., Image-processing projects for an algorithms course

Fotopoulos, S., see Makrogiannis, S.

Fourey, S. \& Malgouyres, R., A digital linking number for discrete curves

Francés, A.R., see Domínguez, E.

Fuh, C.-S., see Chang, M.-C.

Gentili, G.L., Marinilli, M., Micarelli, A. \& Sciarrone, F., Text categorization in an intelligent agent for filtering information on the web

Ghahramani, Z., An introduction to hidden Markov models and Bayesian networks

Goldgof, D., see Powell, M.W.

Grewe, L., Effective computer vision instruction through experimental learning experiences

Guo, J.K., Doermann, D. \& Rosenfeld, A., Forgery detection by local correspondence

Haider, A. Md. \& Kaneko, T. Automated 3D-2D projective registration of human facial images using edge features

He, H. \& Chen, Y.Q., Artificial life for image segmentation

15 (2001) 1311-1327

15 (2001) 967-987

15 (2001) 989-1003

15 (2001) 949-966

15 (2001) 1023-1030

15 (2001) 1311-1327

15 (2001) 347-358

15 (2001) 579-641

15 (2001) 1031-1052

15 (2001) 823-831

15 (2001) 255-267

15 (2001) 223-237

15 (2001) 1201-1212

15 (2001) 789-803

15 (2001) 659-673

15 (2001) 1213-1229

15 (2001) 329-345

15 (2001) 1007-1021

15 (2001) 859-868

15 (2001) 255-267

15 (2001) 1053-1074

15 (2001) 1031-1052

15 (2001) 675-690

15 (2001) 527-549

15 (2001) 9-94

15 (2001) 833-844

15 (2001) 805-821

15 (2001) 579-641

15 (2001) 1263-1276

15 (2001) 989-1003 
Heath, M., see Fink, E.

Hornegger, J. \& Niemann, H., A novel probabilistic model for object recognition and pose estimation

$\mathrm{Hu}, \mathrm{Y}$., see Sun, X.

Huo, C. \& Bhattacharya, P., Content-based indexing of volumetric images using principal component analysis

Iliopoulos, C.S. \& Reid, J.F., Decomposition of partially occluded strings in the presence of errors

Inoue, K., see Okazaki, T.

Ito, A., see Okazaki, T.

Jiang, X., see Yu, K.

Jing, H., see Liu, J.

Jmaiel, M., see Chainbi, W.

Juang, B.H., Introduction: a simple complex in artificial intelligence and machine learning

Kaneko, T., see Haider, A. Md.

Kender, J.R., Visual interfaces to computers: a systems-oriented first course in reliable control via imagery ("visual interfaces")

Kim, J., see Lee, J.J.

Kim, J.H., see Lee, J.J.

Kim, S.I., see Ahn, S.J.

Kovalevsky, V., Curvature in digital 2D images

Kwok, P.C.K., see Fang, B.

Lam, E.C.M., see Tao, Y.

Lee, J.J., Kim, J. \& Kim, J.H., Data-driven design of HMM topology for online handwriting recognition

Lee, K.-L. \& Chen, L.-H., Unsupervised texture segmentation by determining the interior of texture regions based on wavelet transform

Lee, M.-R., 3D shape reconstruction of hybrid reflectance using the LMS algorithm

Lee, S.-W., see Park, H.-S.

Leung, C.H., see Fang, B.

Liao, H.-Y., see Chen, L.-H.

Lin, Z.Y. \& Liu, P., Structural attribute feature code representation and recognition of multifont printed Chinese characters

Liu, C., see Zhong, N.

Liu, J. \& Jing, H., ALIFE: a multiagent computing paradigm for constraint satisfaction problems

Liu, J., Zhong, N., Tang, Y.Y. \& Wang, P.S.P., Introduction

Liu, P., see Lin, Z.Y.

Lu, Z., see Natarajan, P.

Ma, J., A neural network approach to real-time pattern recognition

Makhoul, J., see Natarajan, P.

Makrogiannis, S., Economou, G. \& Fotopoulos, S., A fuzzy dissimilarity function for region based segmentation of color images Malgouyres, R., Editorial

Malgouyres, R., see Fourey, S.

Malgouyres, R., Computing the fundamental group in digital spaces

15 (2001) 859-868

15 (2001) 241-253

15 (2001) 707-721

15 (2001) 1299-1309

15 (2001) 1129-1142

15 (2001) 1143-1165

15 (2001) 1143-1165

15 (2001) 161-176

15 (2001) 475-491

15 (2001) 435-450

15 (2001) 5-7

15 (2001) 1263-1276

15 (2001) 869-884

15 (2001) 107-121

15 (2001) 107-121

15 (2001) 905-919

15 (2001) 1183-1200

15 (2001) 659-673

15 (2001) 1277-1298

15 (2001) 107-121

15 (2001) 1231-1250

15 (2001) 723-734

15 (2001) 91-105

15 (2001) 659-673

15 (2001) 1311-1327

15 (2001) 287-309

15 (2001) 451-473

15 (2001) 475-491

15 (2001) 375-377

15 (2001) 287-309

15 (2001) 43-63

15 (2001) 937-947

15 (2001) 43-63

15 (2001) 255-267

15 (2001) 1005

15 (2001) 1053-1074

15 (2001) 1075-1088 
Marinilli, M., see Gentili, G.L.

15 (2001) 527-549

Marković, M., see Pejnović, P.

15 (2001) 311-327

Marti, U.-V. \& Bunke, H., Using a statistical language model to improve the performance of an HMM-based cursive handwriting recognition system

15 (2001) 65-90

Martin, C.E., see Barber, K.S.

15 (2001) 405-433

Maulik, U., see Bandyopadhyay, S.

Maxwell, B.A., A survey of computer vision education and text resources

McCabe, A., see Caelli, T.

McCane, B., see Novins, K.

Meyer, F., An overview of morphological segmentation

Micarelli, A., see Gentili, G.L.

Moon, J. , see Park, H.-S.

Moreno, A.B., see Sanchez, A.

Müller, S., Eickeler, S. \& Rigoll, G., An integrated approach to shape and color-based image retrieval of rotated objects using hidden Markov models

15 (2001) 269-285

15 (2001) 757-773

15 (2001) 197-221

15 (2001) 775-787

15 (2001) 1089-1118

15 (2001) 527-549

15 (2001) 91-105

15 (2001) 789-803

15 (2001) 223-237

Natarajan, P., Lu, Z., Schwartz, R., Bazzi, I. \& Makhoul, J., Multilingual machine printed OCR

Novins, K. \& McCane, B., Incorporating primary source material into the undergraduate computer vision curriculum

Niemann, H., see Hornegger, J.

Oh, I.-S., see Choi, S.-M.

Ohsuga, S., see Zhong, N.

Ohsuga, S., How can AI systems deal with large and complex problems?

Okada, M., see Chen, Y.

Okazaki, T., Inoue, K., Ito, A. \& Wang, Y., Closure property of space-bounded two-dimensional alternating Turing machines, pushdown automata, and counter automata

Pakhira, M.K., see Bandyopadhyay, S.

Park, H.-S., Sin, B.-K., Moon, J. \& Lee, S.-W., A 2-D HMM method for offline handwritten character recognition

Pejnović, P., Marković, M. \& Stanković, S., Shape classification by moment and autoregressive invariants

Peng, J., see Xiao, Y.

Powell, M.W. \& Goldgof, D., Software toolkit for teaching image processing

Quintero, A., see Domínguez, E.

Rauh, W., see Ahn, S.J.

Reid, J.F., see Iliopoulos, C.S.

Rigoll, G., see Müller, S.

Rosenfeld, A., see Guo, J.K.

Sanchez, A., Velez, J.F., Moreno, A.B. \& Esteban, J.L., Introducing algorithm design techniques in undergraduate digital image processing courses

Sansone, C., Tortorella, F. \& Vento, M., A classification reliability driven reject rule for multi-expert systems

Schüpp, S., see Elmoataz, A.

Schwartz, R., see Natarajan, P.

15 (2001) 43-63

15 (2001) 775-787

15 (2001) 241-253

15 (2001) 949-966

15 (2001) 451-473

15 (2001) 493-525

15 (2001) 967-987

15 (2001) 1143-1165

15 (2001) 269-285

15 (2001) 91-105

15 (2001) 311-327

15 (2001) 347-358

15 (2001) 833-844

15 (2001) 1031-1052

15 (2001) 905-919

15 (2001) 1129-1142

15 (2001) 223-237

15 (2001) 579-641

15 (2001) 789-803

15 (2001) 885-904

15 (2001) 1201-1212

15 (2001) 43-63 
Sciarrone, F., see Gentili, G.L.

15 (2001) 527-549

Serino, L., see Arcelli, C.

Sin, B.-K., see Park, H.-S.

Stanković, S., see Pejnović, P.

15 (2001) 643-657

15 (2001) 91-105

15 (2001) 311-327

Stark, L., see Bowyer, K.

Stevenson, D.E., Image related applications for a core algorithms course

$\mathrm{Su}, \mathrm{M} .$, see Basu, M.

Sun, X., Yang, L., Tang, Y.Y. \& Hu, Y., A new stroke extraction method of Chinese characters

Tang, Y.Y., see Feng, L.

Tang, Y.Y., see Liu, J.

Tang, Y.Y., see Fang, B.

Tang, Y.Y., see Sun, X.

Tang, Y.Y., see Tao, Y.

Tao, Y., Lam, E.C.M. \& Tang, Y.Y., A combination of fractal and wavelet for feature extraction

Tortorella, F., see Sansone, C.

Tougne, L., see Feschet, F.

Tsang, K.M., Recognition of 2D standalone and occluded objects using wavelet transform

Tsang, K.M., Recognition of 3D thin-plate objects using orthogonal AR model

Tse, K.W., see Fang, B.

Tsotsos, J.K., see Ye, Y.

Velez, J.F., see Sanchez, A.

Venkatesh, S., see Bui, H.H.

Vento, M., see Sansone, C.

Wang, P.S.P., see Liu, J.

Wang, Y., see Okazaki, T.

Wang, Y.Y., see Fang, B.

Wang, Z., see Ben-Arie, J.

West, G., see Bui, H.H.

Wild, M., On the idempotency and co-idempotency of the morphological center

Wilson, A.D. \& Bobick A.F., Hidden Markov models for modeling and recognizing gesture under variation

Wong, Y.K., see Fang, B.

Xiao, Y., Ding, M. \& Peng, J., B-spline based stereo for 3D reconstruction of line-like objects using affine camera model

Yang, L., see Sun, X.

Ye, Y. \& Tsotsos, J.K., Knowledge granularity spectrum, action pyramid, and the scaling problem

Yu, K., Jiang, X. \& Bunke, H., Sentence lipreading using hidden Markov model with integrated grammar

Zhong, N., see Liu, J.

Zhong, N., Liu, C. \& Ohsuga, S., Dynamically organizing KDD processes

15 (2001) 753-755

15 (2001) 845-857

15 (2001) 735-752

15 (2001) 707-721

15 (2001) 329-345

15 (2001) 375-377

15 (2001) 659-673

15 (2001) 707-721

15 (2001) 1277-1298

15 (2001) 1277-1298

15 (2001) 885-904

15 (2001) 1007-1021

15 (2001) 691-705

15 (2001) 921-935

15 (2001) 659-673

15 (2001) 379-404

15 (2001) 789-803

15 (2001) 177-195

15 (2001) 885-904

15 (2001) 375-377

15 (2001) 1143-1165

15 (2001) 659-673

15 (2001) 1251-1261

15 (2001) 177-195

15 (2001) 1119-1128

15 (2001) 123-160

15 (2001) 659-673

15 (2001) 347-358

15 (2001) 707-721

15 (2001) 379-404

15 (2001) 161-176

15 (2001) 375-377

15 (2001) 451-473 